\title{
THE
}

3-2-2005

\section{Pressure Dependent Study of the Solid-Solid Phase Change in 38-Atom Lennard-Jones Cluster}

\author{
Dubravko Sabo \\ University of Rhode Island \\ David L. Freeman \\ University of Rhode Island, dfreeman@uri.edu \\ J. D. Doll
}

Follow this and additional works at: https://digitalcommons.uri.edu/chm_facpubs

Terms of Use

All rights reserved under copyright.

\section{Citation/Publisher Attribution}

Sabo, D., Freeman, D. L. \& Doll, J. D. (2005). Pressure Dependent Study of the Solid-Solid Phase Change in 38-Atom Lennard-Jones Cluster. Journal of Chemical Physics, 122, 094716. doi: 10.1063/1.1857521 Available at: http://dx.doi.org/10.1063/1.1857521

This Article is brought to you for free and open access by the Chemistry at DigitalCommons@URI. It has been accepted for inclusion in Chemistry Faculty Publications by an authorized administrator of DigitalCommons@URI. For more information, please contact digitalcommons-group@uri.edu. 


\title{
Pressure dependent study of the solid-solid phase change in 38-atom Lennard-Jones cluster
}

\author{
Dubravko Sabo and David L. Freeman \\ Department of Chemistry, University of Rhode Island, Kingston, Rhode Island 02881 \\ J. D. Doll \\ Department of Chemistry, Brown University, Providence, Rhode Island 02912
}

(Received 29 September 2004; accepted 15 December 2004; published online 2 March 2005)

\begin{abstract}
Phase change phenomena in clusters are often modeled by augmenting physical interaction potentials with an external constraining potential to handle evaporation processes in finite temperature simulations. These external constraining potentials exert a pressure on the cluster. The influence of this constraining pressure on phase change phenomena in 38-atom Lennard-Jones clusters is investigated, and it is demonstrated that modest changes in the parameters of the constraining potential can lead to an order of magnitude change in the constraining pressure. At sufficiently high pressures the solid to solidlike phase change region in the 38-atom Lennard-Jones cluster is completely eliminated. (C) 2005 American Institute of Physics. [DOI: 10.1063/1.1857521]
\end{abstract}

\section{INTRODUCTION}

Small clusters of atoms and molecules have received much attention in recent years ${ }^{1}$ owing to their central role in such diverse areas as homogeneous nucleation and heterogeneous catalysis. In addition to their importance, the physical properties of the clusters themselves are inherently interesting, especially when contrasted with the properties of corresponding bulk materials. An important example of such correspondence is the phenomenon of phase change ${ }^{2}$ where clusters undergo rapid changes in physical properties with respect to their energy in a way that is reminiscent of bulk phase transitions.

One system that has received particular attention owing to its rich phenomenology is the 38-atom Lennard-Jones cluster $\left(\mathrm{LJ}_{38}\right)$. In classical canonical simulations ${ }^{3} \mathrm{LJ}_{38}$ has two phase change regions, a low temperature solid-solid region and a higher temperature solid-liquid region. The phase change regions directly reflect the underlying structure of the complex potential energy surface of $\mathrm{LJ}_{38}$ that is characterized by a double-funnel landscape. ${ }^{4}$

Because small clusters have finite vapor pressures, small Lennard-Jones clusters are usually modeled by a combination of the standard Lennard-Jones interaction potential between the constituent particles, $U_{L J}$, as well as a constraining potential $U_{c}$ defined so that any evaporated particle is reflected back to the main body of the cluster. For LennardJones clusters containing $n$ atoms, the potential energy is then modeled by

$$
U\left(\mathbf{r}_{1}, \mathbf{r}_{2}, \ldots, \mathbf{r}_{n}\right)=U_{L J}\left(\mathbf{r}_{1}, \mathbf{r}_{2}, \ldots, \mathbf{r}_{n}\right)+U_{c}\left(\mathbf{r}_{1}, \mathbf{r}_{2}, \ldots, \mathbf{r}_{n}\right)
$$

with

$$
U_{L J}\left(\mathbf{r}_{1}, \mathbf{r}_{2}, \ldots, \mathbf{r}_{n}\right)=4 \epsilon \sum_{i<j}^{n}\left[\left(\frac{\sigma}{r_{i j}}\right)^{12}-\left(\frac{\sigma}{r_{i j}}\right)^{6}\right]
$$

and

$$
U_{c}\left(\mathbf{r}_{1}, \mathbf{r}_{2}, \ldots, \mathbf{r}_{n}\right)=\sum_{i=1}^{n} u_{c}\left(\mathbf{r}_{i}\right)
$$

In Eq. (2), $\sigma$ and $\epsilon$ are the standard Lennard-Jones length and energy parameters, $\mathbf{r}_{i}$ is the coordinate of particle $i, r_{i j}$ is the distance between particles $i$ and $j$, and several forms for the single particle constraining potential have been used in Eq. (3). We focus on two commonly used forms of the constraining potential. The first, and probably most often applied, is a hard-wall potential first introduced by Lee, Barker, and Abraham, ${ }^{5}$ and defined by

$$
u_{c}^{h w}(\mathbf{r})= \begin{cases}0 & \left|\mathbf{r}-\mathbf{r}_{c m}\right| \leqslant r_{c} \\ \infty & \left|\mathbf{r}-\mathbf{r}_{c m}\right|>r_{c},\end{cases}
$$

where $\mathbf{r}_{c m}$ is the coordinate of the center of mass of the cluster and $r_{c}$ is called the constraining radius. The second form of the constraining potential ${ }^{6}$ is

$$
u_{c}^{c}(\mathbf{r})=\epsilon^{\prime}\left(\frac{\left|\mathbf{r}-\mathbf{r}_{c m}\right|}{r_{c}}\right)^{20},
$$

where $\epsilon^{\prime}$ is an energy parameter and $r_{c}$ is a length parameter that we also call the constraining radius. The continuous form of the constraining potential given in Eq. (5) is often applied in quantum simulations where derivatives of the potential are needed and continuous functions can be advantageous. ${ }^{7,8}$ In simulations on Lennard-Jones systems, it is common to set $\epsilon^{\prime}=\epsilon$, and we make that assignment in the current work.

When either form of the constraining potential is used, the constraining radius parameter must be chosen with care. The constraining potential is artificial, and it is important that the properties of interest in a particular cluster simulation be only weakly dependent on $r_{c}$. Lee, Barker, and Abraham ${ }^{5}$ have shown that the low temperature free energies of interest in their work are only weakly dependent on $r_{c}$, but it is easy to show that other properties can be more sensitive to the 

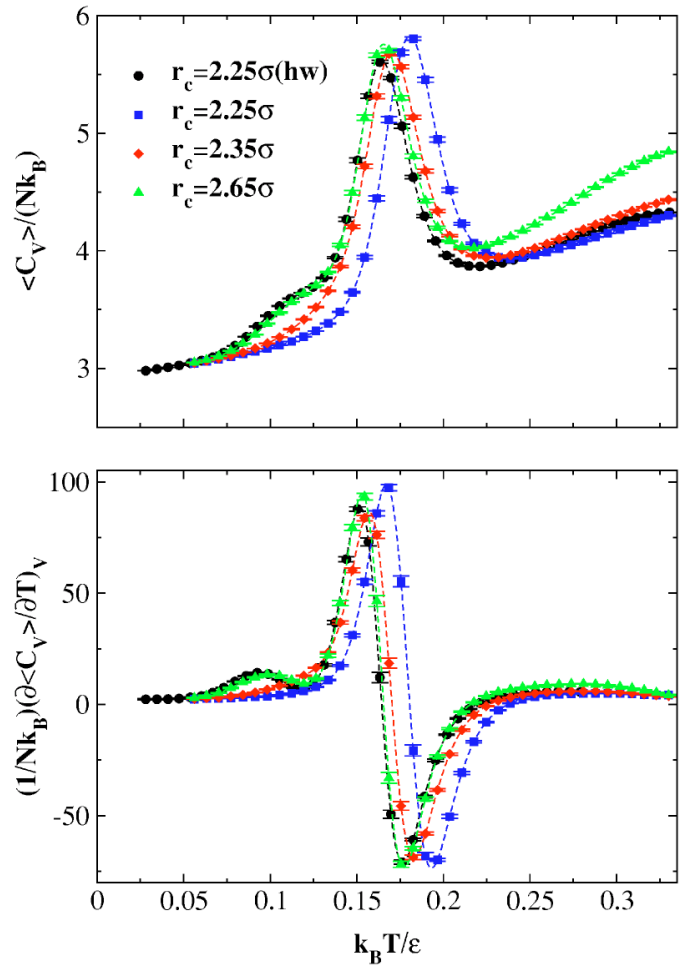

FIG. 1. The heat capacity $C_{V}$ (upper panel) and derivative of the heat capacity $\left(\partial C_{V} / \partial T\right)_{V}$ (lower panel) of $\mathrm{LJ}_{38}$ given in units of $k_{B}$ per particle as a function of temperature for different values of the constraining radius $r_{c}$. Solid circles represent the results obtained by utilizing the hard-wall constraining potential with the constraining radius $r_{c}=2.25 \sigma$. Solid squares, diamonds, and triangles represent the results obtained by utilizing the continuous constraining potential with $r_{c}=2.25 \sigma, r_{c}=2.35 \sigma$, and $r_{c}=2.65 \sigma$, respectively. The error bars correspond to two standard deviations of the mean.

choice of the parameter. It is important to verify for a particular application that the chosen value of $r_{c}$ is sufficiently large so that the properties of interest are not altered in a way that is not physical. On the other hand, as shown elsewhere, ${ }^{9}$ if $r_{c}$ is chosen to be too large, it is difficult to attain ergodicity in a simulation. Consequently, $r_{c}$ must be chosen to be sufficiently small that a simulation is ergodic while ensuring that the calculated properties are not affected significantly.

In previous parallel tempering simulations of $\mathrm{LJ}_{38}$ in both the microcanonical ${ }^{10}$ and canonical ensembles, ${ }^{3}$ the hard-wall constraining potential has been used with $r_{c}$ $=2.25 \sigma$. For the hard-wall constraining potential, this particular choice of constraining radius has been found to meet the sensitive criteria discussed in the previous paragraph. Recently we have noticed that the solid-solid transition does not occur in $\mathrm{LJ}_{38}$ when the continuous form of the constraining potential is used with $r_{c}=2.25 \sigma$. The solid-solid transition does occur if the radius is increased. Evidently, the continuous form of the constraining potential with $r_{c}=2.25 \sigma$ induces sufficient pressure on the system to cause the solid-solid transition to disappear. To see this effect, we examine the data in Fig. 1 that display the constant-volume heat capacity and its derivative as a function of temperature for various values of the constraining radius. Both the constant-volume heat capacity and its derivative are calculated by the standard fluctuation expressions given by Eqs. (8) and (9) in Ref. 8 .
The constant-volume heat capacity curve marked by the solid circles is obtained by utilizing the hard-wall constraining potential with the constraining radius $r_{c}=2.25 \sigma$, while all other curves are obtained by utilizing the continuous constraining potential. By decreasing the constraining radius of the continuous constraining potential from $r_{c}=2.65 \sigma$ to $r_{c}$ $=2.25 \sigma$, the signature of the solid-solid transition gradually disappears.

Motivated by the observed sensitivity of the existence of the solid-solid change to $r_{c}$, in this work we investigate the effect of pressure on the solid-solid phase change in $\mathrm{LJ}_{38}$ using Eq. (5) for the constraining potential. We focus on the continuous form of the constraining potential, because of the importance of that form of the constraining potential to quantum simulations. There have been previous classical ${ }^{11,12}$ and quantum ${ }^{13}$ investigations of cluster phase change phenomena as a function of pressure using the isothermal-isobaric ensemble. As discussed elsewhere, ${ }^{14-19}$ the application of the isothermal-isobaric ensemble to small system like clusters is subtle. The subtleties can lead to errors in the computed thermodynamic properties, with the errors becoming small in the thermodynamic limit. In the current work, we avoid these ambiguities by defining a constraining pressure $p_{c}$ in the canonical ensemble and using canonical simulations to calculate the defined constraining pressure at different values of the constraining radius and temperature. We demonstrate the sensitivity of the existence of the solid-solid phase change to the calculated constraining pressure by examining the effect of $p_{c}$ to the inherent structure distribution from the higher energy basin.

The contents of the remainder of this paper are as follows. In the following section we present the theoretical methods used including a definition of the connection between the constraining pressure on the cluster and its relation to the constraining volume that we must define when the continuous form of the constraining potential is used. In Sec. III we present the results of numerical simulations on $\mathrm{LJ}_{38}$ and we discuss our results in Sec. IV.

\section{THEORY}

\section{A. The constraining volume}

We begin by developing an expression for the constraining volume when Eq. (5) is used for the constraining potential. If we use Eq. (4) for the constraining potential, the obvious choice, $V_{c}=(4 / 3) \pi r_{c}^{3}$, is appropriate. However, as discussed in the Introduction, we have found for a given $r_{c}$ that the effective volume when Eq. (5) is used for the constraining potential appears to be smaller than when Eq. (4) is used, and the standard expression for the volume of a sphere does not reflect the decreased volume associated with the continuous constraining potential. We expect the effective volume associated with the continuous form of the constraining potential to be temperature dependent, because particles should fill more space as their energy is increased. To find a definition of the constraining volume that has the expected qualitative features, we notice that the single particle configurational integral at temperature $T$ for the hard-wall constraining potential gives 


$$
z_{c, h w}=\int d^{3} r e^{-\beta u_{c}^{h w}(\mathbf{r})}=\frac{4}{3} \pi r_{c}^{3},
$$

which is just the volume of a sphere of radius $r_{c}$. As usual, in Eq. (6), $\beta=1 / k_{B} T$ with $k_{B}$ the Boltzmann constant. In analogy with Eq. (6), we define the constraining volume associated with Eq. (5) using the expression

$$
\begin{aligned}
V_{c} & =\int d^{3} r e^{-\beta u_{c}^{c}(\mathbf{r})} \\
& =4 \pi \int_{0}^{\infty} d r r^{2} e^{-\beta \epsilon r^{20 / r_{c}^{20}}} \\
& =\frac{\pi r_{c}^{3}}{5(\beta \epsilon)^{3 / 20}} \Gamma\left(\frac{3}{20}\right) .
\end{aligned}
$$

If we calculate the ratio of this constraining volume to the volume of the hard-wall potential; i.e., $(4 / 3) \pi r_{c}^{3}$, we find

$$
R=\frac{V_{c}}{(4 / 3) \pi r_{c}^{3}}=\frac{3 \Gamma(3 / 20)}{20(\beta \epsilon)^{3 / 20}} .
$$

At low temperatures (large $\beta$ ) $R$ is less than 1 and we expect the effective pressure of the continuous constraining potential to exceed that from the hard-wall potential. At high temperatures (small $\beta$ ) the ratio $R$ exceeds 1 and we have a decreased effective constraining pressure. Using MATHEMATICA $^{20}$ we find $R=1$ at $\beta \epsilon \cong 0.63$ or in reduced units $k_{B} T / \epsilon=1.59$. This reduced temperature is large compared to the typical temperatures explored in our investigations and very large compared to the reduced temperature of the solid-solid phase change in $\mathrm{LJ}_{38}$. Consequently, at our expected working temperatures, the defined constraining volume associated with the continuous form of the constraining potential is less than that of the hard-wall potential.

\section{B. The constraining pressure}

Given our defined constraining volume, we next need a definition of the constraining pressure associated with the continuous constraining potential. In analogy with the expression for the pressure of a system in terms of the canonical partition function $Q(T, V, N)$ in the canonical ensemble

$$
p=k_{B} T\left(\frac{\partial \ln Q}{\partial V}\right)_{N, T},
$$

we define the constraining pressure in the canonical ensemble with the expression

$$
p_{c}=k_{B} T\left(\frac{\partial \ln z}{\partial V_{c}}\right)_{n, T},
$$

where $z$ is the configurational integral

$$
z=\int d^{3 n} r e^{-\beta U\left(\mathbf{r}_{1}, \mathbf{r}_{2}, \ldots, \mathbf{r}_{n}\right)} .
$$

Using Eq. (12) we derive the relation between our defined constraining pressure and defined constraining volume,

$$
\begin{aligned}
p_{c} & =\frac{k T}{z}\left(\frac{\partial}{\partial V_{c}} \int d^{3 n} r e^{-\beta\left[U_{L J}\left(\mathbf{r}_{1}, \mathbf{r}_{2}, \ldots, \mathbf{r}_{n}\right)+U_{c}\left(\mathbf{r}_{1}, \mathbf{r}_{2}, \ldots, \mathbf{r}_{n}\right)\right]}\right)_{n, T} \\
& =-\frac{1}{z} \int d^{3 n} r\left(\frac{\partial U_{c}}{\partial V_{c}}\right)_{n, T} e^{-\beta U\left(\mathbf{r}_{1}, \mathbf{r}_{2}, \ldots, \mathbf{r}_{n}\right)} .
\end{aligned}
$$

Using

$$
\left(\frac{\partial U_{c}}{\partial V_{c}}\right)_{n, T}=\left(\frac{\partial U_{c}}{\partial r_{c}}\right)_{n, T}\left(\frac{\partial r_{c}}{\partial V_{c}}\right)_{n, T},
$$

we find

$$
\left(\frac{\partial U_{c}}{\partial V_{c}}\right)_{n, T}=-\frac{100(\beta \epsilon)^{3 / 20}}{3 \pi r_{c}^{3} \Gamma(3 / 20)} U_{c}
$$

so that

$$
\begin{aligned}
p_{c} & =\frac{1}{z} \int d^{3 n} r \frac{100(\beta \epsilon)^{3 / 20}}{3 \pi r_{c}^{3} \Gamma(3 / 20)} U_{c} e^{-\beta U} \\
& =\frac{100(\beta \epsilon)^{3 / 20}}{3 \pi r_{c}^{3} \Gamma(3 / 20)}\left\langle U_{c}\right\rangle \\
& =\frac{20\left\langle U_{c}\right\rangle}{3 V_{c}} .
\end{aligned}
$$

\section{RESULTS}

\section{A. Computational details}

We have carried out parallel tempering Monte Carlo simulations ${ }^{21-23}$ using nine constraining radii ranging from $2.20 \sigma$ to $2.65 \sigma$. The continuous form of the constraining potential has been used. For a given constraining radius, distinct temperatures, equally separated by $\Delta T=7.022$ $\times 10^{-3} \epsilon / k_{B}$, have been generated in the range from $T$ $=0.0563 \epsilon / k_{B}$ to $T=0.3301 \epsilon / k_{B}$. Each temperature is associated with a different processor that runs its own replica of the system and its own stream. A stream is an independent and uncorrelated sequence of random numbers that can be generated simultaneously on multiple processors. In the present work, we have implemented the scalable parallel random number generator. ${ }^{24,25}$ The above choice of temperature gap $\Delta T$ is adequate because the acceptance probability for swaps (exchange of configurations) between the adjacent temperatures is larger than 0.5 for all streams and for all simulations performed.

The Monte Carlo simulations are performed as follows. For each stream, a random walk is carried out through configuration space including moves in the particle coordinates using the Metropolis algorithm ${ }^{26}$ where the acceptance probability for trial moves is given by

$$
\operatorname{acc}\left(\mathbf{r}_{i} \rightarrow \mathbf{r}_{j}\right)=\min \left(1, \exp \left\{-\beta\left[U\left(\mathbf{r}_{j}\right)-U\left(\mathbf{r}_{i}\right)\right]\right\}\right) .
$$

We define a pass as the minimal set of Monte Carlo attempted moves over all particles in the system. A set of 100000 passes defines a block. The size of the block is sufficiently large that the block averages are independent. The 


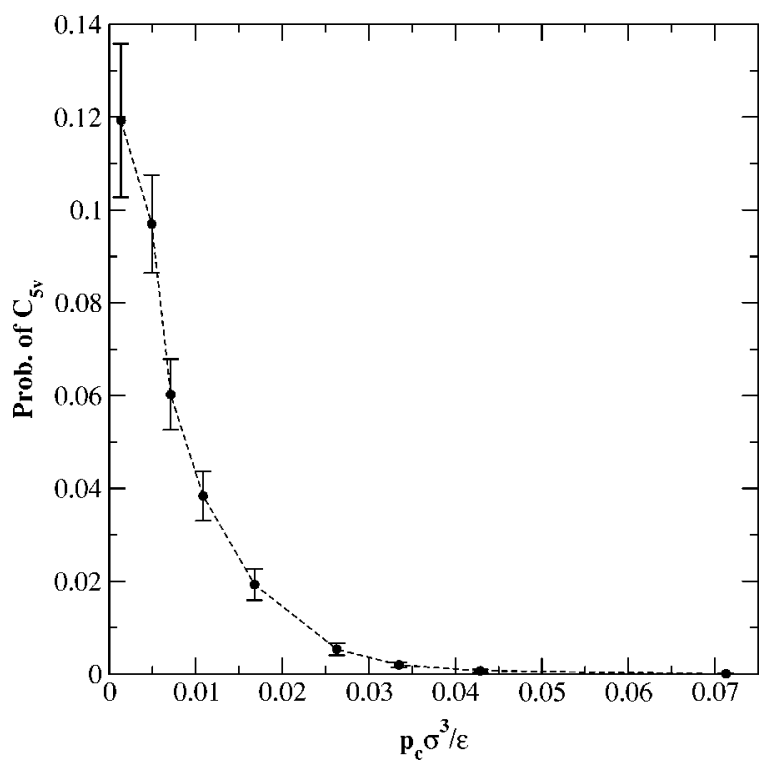

FIG. 2. The probability of observing the icosahedral structures as a function of the constraining pressure. The probability is obtained by quenching the configurations sampled from the parallel tempering Monte Carlo simulations at the temperature of solid-solid transition, $T=0.1 \epsilon / k_{B}$. The error bars are two standard deviations of the mean.

simulations are divided in two stages; an equilibration stage that consists of 400 blocks and an accumulation stage that consists of 1000 blocks per temperature.

An exchange of configurations between streams at adjacent temperatures $(i$ and $j$ ) has been attempted every ten passes and it has been accepted or rejected according to acceptance probability

$$
\operatorname{acc}(i \rightarrow j)=\min \left\{1, \exp \left[-\left(\beta_{i}-\beta_{j}\right)\left(U_{j}-U_{i}\right)\right]\right\} .
$$

A stream at any given temperature attempts a swap of configurations with a stream at adjacent lower and higher temperature in succession. Because of this swapping strategy, the streams at minimum and maximum temperatures are involved in swaps only every 20 passes. The simulations at each radius less than $2.65 \sigma$ have been initiated from the equilibrated configurations taken from the study of the system at the next highest radius. These starting configurations have been then further equilibrated with $40 \times 10^{6}$ additional parallel tempering Monte Carlo passes prior to the accumulation of data. The error bars of all results displayed in this work represent two standard deviations of the mean.

\section{B. Numerical results}

The solid-solid transition corresponds to the structural transformation between a truncated octahedral (a global minimum with $O_{h}$ symmetry) and icosahedral structure(s) (a second lowest lying minimum with $C_{5 v}$ symmetry or any low-lying minima associated with that basin). ${ }^{4}$ Figure 2 shows the probability of observing the icosahedral-based structures as a function of the constraining pressure. The constraining pressure has been calculated using Eq. (20), and the probability has been estimated by performing minimization or quenching of the parallel tempering Monte Carlo sampled configurations to their nearest inherent structures.
The quenching of the configurations has been achieved by implementing the conjugate gradient method using the algorithm given in Numerical Recipes. ${ }^{27}$ We have carried out four simulations for each value of the constraining radius and saved 100000 configurations at the temperature at which the solid-solid transition occurs, $T=0.1 \epsilon / k_{B}$. Quenching of the configurations have yielded probability of observing the icosahedral structure. The final probability has been obtained by averaging over the set of four probability values. At the lower constraining pressures the system dwells in the icosahedral basin with probability of about $10 \%$. At these constraining pressures the solid-solid transition is still visible in the heat capacity (and the derivative of the heat capacity) curves. The probability drops significantly at higher constraining pressures and eventually drops to zero at $p_{c} \cong 4.0$ $\times 10^{-2} \epsilon / \sigma^{3}$. For these constraining pressures the signature of the solid-solid transition disappears from the heat capacity curve.

\section{DISCUSSION}

To study phase change phenomena in clusters it is common to introduce an external constraining potential to handle the evaporation events inherent in finite temperature simulations. The solid to solidlike phase change in $\mathrm{LJ}_{38}$ is an example of a cluster phase change that has received much attention principally owing to difficulties in simulating systems with complex potential surfaces. Because the solid to solidlike phase change in $\mathrm{LJ}_{38}$ occurs at temperatures well below the melting phase change region, the influence of the size of the external constraining potential has not been a major concern. In the current work we have demonstrated that the existence of the solid to solidlike phase change region is remarkably sensitive to the radius of the external constraining potential. When continuous forms of the constraining potential are used, changes of about $10 \%$ in the constraining radius can result in approximately an order of magnitude change in the constraining pressure. By studying the population of inherent structures in the transition region as well as the heat capacity, we have demonstrated that such pressure changes can completely eliminate any evidence of the solid to solidlike phase change.

The results of the current study highlight the importance of examining carefully the influence of artificial constraining potentials on observed thermodynamic properties. The effect of the constraining parameters (e.g., the constraining radius) on the thermodynamic properties must be evaluated over the entire temperature range examined in a calculation. We have demonstrated that even presumably low temperature phenomena can be affected by the forces arising from the constraining potential which is only an artifact of how the calculation is performed.

\section{ACKNOWLEDGMENTS}

This work was supported in part by National Science Foundation Grant Nos. CHE-0095053 and CHE-0131114.

${ }^{1}$ D. L. Freeman and J. D. Doll, Annu. Rev. Phys. Chem. 47, 43 (1996).

${ }^{2}$ R. S. Berry, T. L. Beck, and H. Davis, Adv. Chem. Phys. 70, 75 (1988).

${ }^{3}$ J. P. Neirotti, F. Calvo, D. L. Freeman, and J. D. Doll, J. Chem. Phys. 112, 
10340 (2000).

${ }^{4}$ J. P. K. Doye, M. A. Miller, and D. J. Wales, J. Chem. Phys. 110, 6896 (1999).

${ }^{5}$ J. K. Lee, J. A. Barker, and F. F. Abraham, J. Chem. Phys. 58, 3166 (1973).

${ }^{6}$ D. L. Freeman and J. D. Doll, J. Chem. Phys. 80, 5709 (1984).

${ }^{7}$ C. Predescu, D. Sabo, J. D. Doll, and D. L. Freeman, J. Chem. Phys. 119, 12119 (2003).

${ }^{8}$ D. Sabo, C. Predescu, J. D. Doll, and D. L. Freeman, J. Chem. Phys. 121, 856 (2004).

${ }^{9}$ J. P. Neirotti, D. L. Freeman, and J. D. Doll, Phys. Rev. E 62, 7445 (2000).

${ }^{10}$ F. Calvo, J. P. Neirotti, D. L. Freeman, and J. D. Doll, J. Chem. Phys. 112, 10350 (2000).

${ }^{11}$ W. Ortiz, A. Perlloni, and G. E. López, Chem. Phys. Lett. 298, 66 (1998).

${ }^{12}$ M. C. Vicéns and G. E. López, Phys. Rev. A 62, 030203 (2000).

${ }^{13}$ G. E. López, J. Chem. Phys. 117, 2225 (2002).

${ }^{14}$ P. Attard, J. Chem. Phys. 103, 9884 (1995).
${ }^{15}$ G. J. M. Koper and H. Reiss, J. Phys. Chem. 100, 422 (1996).

${ }^{16}$ D. S. Corti and G. Soto-Campos, J. Chem. Phys. 108, 7959 (1998).

${ }^{17}$ D. S. Corti, Phys. Rev. E 64, 016128 (2001).

${ }^{18}$ K.-K. Han and H. S. Son, J. Chem. Phys. 115, 7793 (2001).

${ }^{19}$ D. S. Corti, Mol. Phys. 100, 1887 (2002).

${ }^{20}$ S. Wolfram, The Mathematica Book (Cambridge University Press, New York, 1999).

${ }^{21}$ E. Marinari and G. Parisi, Europhys. Lett. 19, 451 (1992).

${ }^{22}$ C. J. Geyer and E. A. Thompson, J. Am. Stat. Assoc. 90, 909 (1995).

${ }^{23}$ M. Falcioni and M. Deem, J. Chem. Phys. 110, 1754 (1999).

${ }^{24}$ A. Srinivasan, D. M. Ceperley, and M. Mascagni, Adv. Chem. Phys. 105, 13 (1999).

${ }^{25}$ URL http://sprng.cs.fsu.edu/Science/CMP/RNG-home.html

${ }^{26} \mathrm{M}$. Kalos and P. Whitlock, Monte Carlo Methods (Wiley, New York, 1986).

${ }^{27}$ W. H. Press, S. A. Teukolsky, W. T. Vetterling, and B. P. Flannery, Numerical Recipes, 2nd ed. (Cambridge University Press, Cambridge, 1992). 
The Journal of Chemical Physics is copyrighted by the American Institute of Physics (AIP). Redistribution of journal material is subject to the AIP online journal license and/or AIP copyright. For more information, see http://ojps.aip.org/jcpo/jcpcr/jsp 